Document downloaded from:

http://hdl.handle.net/10251/154794

This paper must be cited as:

Fuentes, M.; Mi, D.; Chen, H.; Garro, E.; Carcel, JL.; Vargas, D.; Mouhouche, B.... (2019). Physical Layer Performance Evaluation of LTE-Advanced Pro Broadcast and ATSC 3.0 Systems. IEEE Transactions on Broadcasting. 65(3):477-488.

https://doi.org/10.1109/TBC.2018.2866778

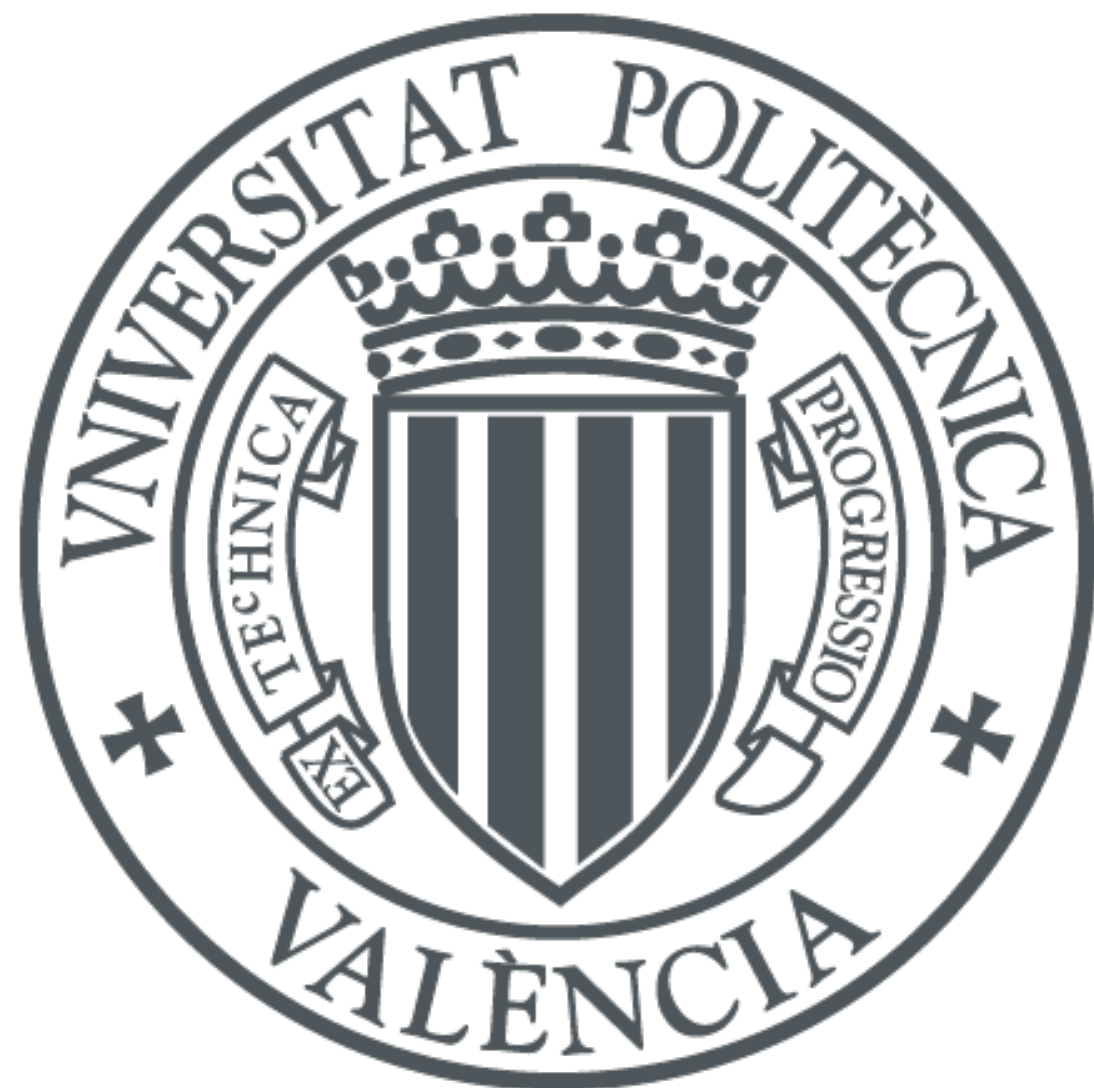

The final publication is available at

https://doi.org/10.1109/TBC.2018.2866778

Copyright Institute of Electrical and Electronics Engineers

Additional Information

(C) 2019 IEEE. Personal use of this material is permitted. Permissíon from IEEE must be obtained for all other uses, in any current or future media, including reprinting/republishing this material for advertisíng or promotional purposes, creating new collective works, for resale or redistribution to servers or lists, or reuse of any copyrighted component of this work in other works. 


\title{
Physical Layer Performance Evaluation of LTE-Advanced Pro Broadcast and ATSC 3.0 Systems
}

\author{
Manuel Fuentes, De Mi, Hongzhi Chen, Eduardo Garro, Jose Luis Carcel, David Vargas, Belkacem Mouhouche
} and David Gomez-Barquero

\begin{abstract}
This work provides a detailed performance analysis of the physical layer of two state-of-the-art point-to-multipoint (PTM) technologies: evolved Multimedia Broadcast Multicast Services (eMBMS) and Advanced Television Systems Committee - Third Generation (ATSC 3.0). The performance of these technologies is evaluated and compared using link-level simulations, considering relevant identified scenarios. A selection of Key Performance Indicators (KPI) for the International Mobile Telecommunications 2020 (IMT-2020) evaluation process has been considered. Representative use cases are also aligned to the test environments as defined in the IMT-2020 evaluation guidelines. It is observed that ATSC 3.0 outperforms both eMBMS solutions, i.e. MBMS over Single Frequency Networks (MBSFN) and Single-Cell PTM (SC-PTM) in terms of spectral efficiency, peak data rate and mobility, among others. This performance evaluation serves as a benchmark for comparison with a potential 5G PTM solution.
\end{abstract}

Index Terms-Benchmark, point-to-multipoint, eMBMS, MBSFN, SC-PTM, ATSC 3.0, broadcasting.

\section{INTRODUCTION}

$\mathbf{P}$ OINT-TO-MULTIPOINT (PTM) communications are the only technology enabling the delivery of the same content to a practically infinite number of users simultaneously, using just a fixed amount of resources for a given coverage area. Traditionally, PTM transmissions have been used to deliver linear content (such as TV or radio) through Digital Terrestrial Television (DTT) systems. Many first-generation DTT systems are nowadays in place over the world, such as Advanced Television Systems Committee (ATSC) [1] in North America, Integrated Services Digital Broadcasting Terrestrial (ISDB-T) [2] in Japan and South America or Digital Terrestrial Multimedia Broadcast (DTMB) [3] in China.

Manuscript submitted May 30, 2018. This work was supported in part by the European Commission under the 5GPPP project 5G-Xcast (H2020-ICT2016-2 call, grant number 761498). The views expressed in this contribution are those of the authors and do not necessarily represent the project. Parts of this paper have been partially published in [11].

M. Fuentes was with Samsung Electronics R\&D UK, Staines-upon-Thames, TW18 4QE, United Kingdom and is now with Universitat Politecnica de Valencia, Valencia, 46022, Spain (e-mail: mafuemue@iteam.upv.es).

D. Mi and H. Chen are with the University of Surrey, Guildford, GU2 7XH, United Kingdom (e-mail: \{d.mi, hongzhi.chen\}@surrey.ac.uk).

E. Garro and D. Gomez-Barquero are with Universitat Politecnica de Valencia, Valencia, 46022, Spain (e-mail: \{edgarcre, dagobar\}@iteam.upv.es).

J. L. Carcel and B. Mouhouche are with Samsung Electronics R\&D UK, Staines-upon-Thames, TW18 4QE, United Kingdom (e-mail: jose.cervera, b.mouhouche@samsung.com).

D. Vargas is with the BBC R\&D, London, W12 7SB, United Kingdom (email: david.vargas@bbc.co.uk).
Among these technologies utilized in many countries, Digital Video Broadcasting - Terrestrial (DVB-T) is the most widely implemented DTT standard in the world [4]. Its evolution, DVB - Second Generation Terrestrial (DVB-T2) [5], provides a 50\% increase of spectral efficiency compared to DVB-T and introduces new technologies such as the use of Low-Density Parity Check (LDPC) codes or higher orders of constellation, using 256 symbols with Quadrature Amplitude Modulation (QAM). Today, the state-of-the-art DTT standard is ATSC Third Generation (ATSC 3.0) [6]. ATSC 3.0 provides better performance than DVB-T2 in terms of carrier-to-noise ratio (CNR) and shortens the gap to the Shannon limit, thanks to the use of more efficient constellations and very robust coding rates (CR), the aggregation of multiple radio-frequency (RF) carriers or the combined provision of fixed and mobile services through the use of non-orthogonal multiplexing techniques.

DTT systems were originally developed to support mainly fixed rooftop reception. Despite the efforts to develop mobile DTT standards such as DVB - Handheld (DVB-H) [7] or DVB - Next Generation Handheld (DVB-NGH) [8], the lack of market limited their success. In parallel, the Third Generation Partnership Project (3GPP) standardization forum developed the fourth generation (4G) standard Long Term Evolution (LTE) to provide high-speed mobile broadband for handheld services through unicast. LTE also adopted the use of evolved Multimedia Broadcast Multicast Services (eMBMS) in Release (Rel-) 9 [9] to deliver mobile video through multicast and broadcast. Today, the state-of-the-art specification for PTM is LTE-Advanced Pro Rel-14, which has included additional requirements to deliver linear services to both mobiles and fixed rooftop receivers.

Since its introduction, eMBMS has gone through a very significant set of enhancements [10]. For instance, it introduced new physical, transport and logical channels in the specification to enable MBMS over Single Frequency Networks (MBSFN). Although LTE-Advanced Pro Rel-14 carries a long legacy because of the backward-compatible design philosophy of LTE, it is indeed very different from Rel-9. The main novelty regarding PTM up to now is the use of Single-Cell PTM (SC-PTM), introduced in Rel-13 to increase the resource allocation flexibility by multiplexing broadcast and unicast data on the same physical channel. Rel-14 also included several changes to the physical layer in MBSFN, such as the use of new numerologies $7.5 \mathrm{kHz}$ and $1.25 \mathrm{kHz}$ (the first option was included in previous releases but could not 

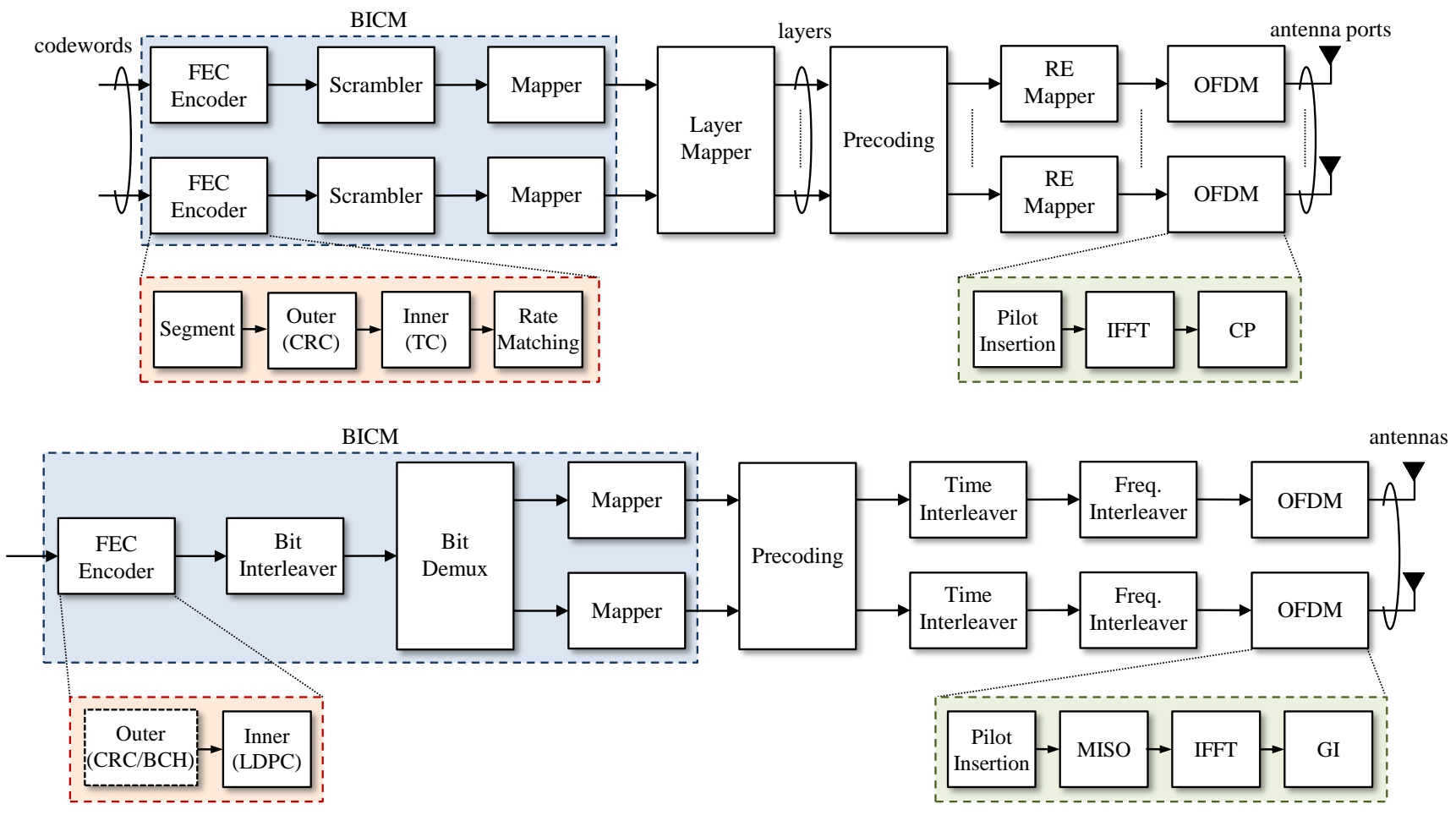

Fig. 1. LTE-Advanced Pro eMBMS (top) and ATSC 3.0 (bottom) physical layer transmitter block diagram.

be used since there was no signalling associated), the use of a Cell Acquisition Subframe (CAS) to allow synchronization for receive only devices or the increase of PTM capacity by allocating all subframes in one frame for broadcast. Note that this is based on the unicast procedure for cell acquisition, synchronization and basic information. Therefore, the analysis of CAS is out of the scope of this work.

Motivated by our prior investigation in [10], this paper aims at providing a comprehensive performance evaluation of the PTM technologies as previously defined, i.e., ATSC 3.0 and eMBMS, utilizing as reference the Key Performance Indicators (KPI) and methodologies defined by the International Telecommunications Union (ITU) for the International Mobile Telecommunication - 2020 (IMT-2020) evaluation process of candidate radio interface technologies [12]. Note that specific results for LTE were partially published in [11], but the present work covers a wider range of use cases. It provides an extensive evaluation based on inspection, analysis and linklevel simulations for a large number of channel models and scenarios.

The results in this paper provide a gap analysis between state-of-the-art PTM technologies and serve as a benchmark for a potential fifth generation $(5 \mathrm{G})$ broadcast/multicast solution [14]. In fact, 3GPP started in March 2017 the normative work for 5G in Rel-15, also known as New Radio (NR), focusing on a point-to-point network infrastructure solution. 3GPP Rel-16 work starts in 2018 and it targets the IMT2020 submission. The 3GPP in [13] has identified, amongst other items, flexible broadcast/multicast service as a basic capability for the $5 \mathrm{G}$ system and sets out a list of potential requirements. However, the support of broadcast and multicast capabilities is currently envisioned for evaluation in Rel-16 or Rel-17 due to the very tight schedule of 5G NR and the high workload in 3GPP. This work provides valuable insights into the physical layer design for practical PTM systems, revealing limitations and potential improvements of the state-of-the-art PTM technologies in this regard.

The rest of the paper is organized as follows. First, the technologies under evaluation are described in Section II. Section III presents the methodology. Section IV provides a complete analysis and defines the current limitations found. Then, both ATSC 3.0 and eMBMS are evaluated through linklevel simulations in Section IV. Finally, Section V summarizes the findings of the investigations carried out and discusses the main areas of potential improvement towards the development of technical solutions in the future.

\section{Physical Layer Overview}

This section presents a physical layer overview of the two technologies considered in this study, i.e. LTE-Advanced Pro eMBMS and ATSC 3.0. Descriptions are focused on transmission, since receiver implementations depend on manufacturers.

\section{A. LTE-Advanced Pro eMBMS}

LTE-Advanced Pro eMBMS Rel-14 is the latest standardized LTE PTM technology. Fig. 1 (top) depicts the generic transmitter block diagram. A single or two transport blocks (TB) containing the data can be transmitted. The number of codewords is directly related to the TBs and is always the same or lower than the number of layers and antenna 
ports. Data bits are encoded using a combination of error detection, error correction and rate matching. First, a Cyclic Redundancy Check (CRC) bit sequence is attached to each TB. If the TB size is larger than the maximum code block (CB) size of 6144 bits, the input data sequence is then segmented and an additional CRC sequence is attached to each CB. The output bits for a given $\mathrm{CB}$ are then coded using a turbo code (TC) with CR 1/3. Next, rate matching is performed so that the bits of each $\mathrm{CB}$ are interleaved, circularly buffered and punctured or repeated, depending on the available resources, to provide the specific CR related to the input Modulation and Coding Scheme (MCS) selected. Bits generated are then concatenated, scrambled and split into groups of bits depending on the modulation order and mapped to constellation symbols. Constellations available in eMBMS are Quadrature Phase Shift Keying (QPSK), 16QAM, 64QAM and 256QAM. The complex-valued modulation symbols are next mapped onto one or several layers and precoded for transmission on the antenna ports. Complex symbols are then located in the resource elements available in the corresponding subframe, and modulated to transmit using an Orthogonal Frequency-Division Multiplexing (OFDM) signal. Finally, a cyclic prefix (CP) with specific duration is inserted at the beginning of each OFDM symbol.

LTE-Advanced Pro Rel-14 provides a wide set of possible bandwidth allocations, i.e. 1.4, 3, 5, 10 and $20 \mathrm{MHz}$. Additionally, it permits from Rel-13 a maximum carrier aggregation of $32 \mathrm{RF}$ carriers. The maximum aggregated bandwidth is therefore $640 \mathrm{MHz}$, although none commercial network with this bandwidth has been deployed up to now. The physical layer of eMBMS has two options whether the transmission is done over a single cell (SC-PTM) or over multiple cells in a synchronised manner by SFN transmissions (MBSFN).

1) Single-Cell Point-to-Multipoint: The SC-PTM solution aims at increasing the resource allocation flexibility for PTM deployments. It allows a single cell to broadcast content to a group of users on the Physical Downlink Shared Channel (PDSCH), which is used for unicast transmissions. Sharing a physical channel also implies to use the same carrier spacing of $15 \mathrm{kHz}$. With SC-PTM, both normal CP (5.2 $\mu$ s first symbol and $4.7 \mu$ s the rest) and extended $\mathrm{CP}(16.7 \mu \mathrm{s})$ are available to use. The use of a Multiple-Input Multiple-Output (MIMO) configuration with up to four transmitter and receiver antennas is permitted in this case.

2) MBMS over Single Frequency Networks: MBSFN deployments consist of a group of cells that perform completely synchronized transmission, reducing inter-cell interference for the broadcast service within the given area. The trade-off here comes in flexibility. On the one hand MBSFN transmissions use a specific Physical Multicast Channel (PMCH) occupying the entire bandwidth, but on the other hand there is fixed resource allocation with rigid parameters. MBSFN can be configured with three carrier spacing values of $15,7.5$ and $1.25 \mathrm{kHz}$, related to extended CP lengths of 16.7, 33.3 and $200 \mu$ s respectively. Due to the SFN transmission, MBSFN uses a more dense reference signal pattern than the one used for unicast. It is important to note that MIMO techniques that provide spatial multiplexing gain are not defined for MBSFN, and therefore a single codeword is only transmitted.

\section{B. ATSC 3.0}

Fig. 1 (bottom) presents the ATSC 3.0 transmitter block diagram. As Fig. 1 shows, up to two antennas are permitted with this technology. The input stream is encoded using a combination of an optional outer Bose-Chaudhuri-Hocquenghem $(\mathrm{BCH})$ or $\mathrm{CRC}$ code and an inner LDPC code [15]. There are two different LDPC code lengths defined, i.e. 16200 bits (short codes) and 64800 bits (long codes). For $2 \times 2$ MIMO, only the long code is specified. Note that ATSC 3.0 permits to use a set of 12 coding rates from $2 / 15$ to $13 / 15$, with $1 / 15$ step.

Output bits from the encoder are bit interleaved (BIL). In case of MIMO transmissions, the bit demultiplexer distributes the bits from the BIL into the two separated mappers, one per transmitter antenna. The input to the constellation mapping block in each stream is a Forward Error Correction (FEC) frame and the output is a FEC block. Bits are mapped to complex-valued symbols using Non-Uniform Constellations (NUC). In addition to QPSK, ATSC 3.0 implements 2D-NUCs with 16, 64 and 256 symbols. 1D-NUCs with 1024 and 4096 symbols can be also used with long LDPC codes. In total, there are 72 and 48 modulation and CR combinations for long and short codes respectively. Note that only 46 and 29 combinations are mandatory to be implemented. This decision was taken in order to reduce the number of combinations to a practical number while keeping a good flexibility [16] [17].

A MIMO precoding is applied to the mapped FEC blocks if needed. FEC blocks coming from the precoder are then time (TIL) and frequency interleaved (FIL) in order to provide additional time and frequency diversity respectively. Finally, the OFDM waveform is generated by inserting pilot subcarriers, applying the inverse Fast Fourier Transform (FFT) and inserting the $\mathrm{CP}$, here called Guard Interval (GI). ATSC 3.0 provides a single $6 \mathrm{MHz}$ bandwidth allocation that can be extended to $12 \mathrm{MHz}$ when using $2 \mathrm{RF}$ carriers via channel bonding to achieve greater data rates.

\section{Evaluation Methodology}

The IMT-2020 evaluation process defines technical performance requirements for main usage scenarios and their corresponding evaluation methodology for candidate radio interface technologies [18]. The methodology in this paper is structured around the different types of evaluation considered in the IMT-2020 guidelines [12]. KPIs extracted from these guidelines have been also selected in order to evaluate the considered PTM wireless technologies. Moreover, this work has defined two additional KPIs to better assess the overall performance of PTM transmissions. These two KPIs defined have been widely used in the standardization of DTT systems such as DVB-T2 or ATSC 3.0.

Table I presents a summary of the high-level assessment methods used per KPI. In this work, two evaluation procedures are considered. The first procedure is a mathematical analysis. The evaluation is based on calculations that use technical 
TABLE I

HIGH-LEVEL ASSESSMENT METHOD PER KPI

\begin{tabular}{cccc}
\hline \hline KPI & Units & Method & Origin \\
\hline Peak data rate & $\mathrm{bit} / \mathrm{s}$ & Analysis & IMT-2020 \\
Peak spectral efficiency & $\mathrm{bit} / \mathrm{s} / \mathrm{Hz}$ & Analysis & IMT-2020 \\
Peak BICM spectral efficiency & $\mathrm{bpc}$ & Analysis & DTT \\
BICM spectral efficiency & $\mathrm{bpc}$ & Link-level & DTT \\
Mobility & $\mathrm{km} / \mathrm{h}$ & Link-level & IMT-2020 \\
\hline \hline
\end{tabular}

information. The second procedure is carried out through linklevel simulations. This method is applied to KPIs that are heavily dependent on the instantaneous network conditions.

\section{A. Peak Data Rate}

The first KPI evaluated through analysis is the peak data rate, expressed in bit/s. It is calculated using:

$$
\gamma_{p}=\frac{N_{\text {data }}}{T}
$$

where $N_{\text {data }}$ is the maximum number of data bits transmitted in a period of time $T$. The peak data rate calculation depends on the technology under study. For LTE-Advanced Pro, $N_{\text {data }}$ is the maximum TB size (TBS) delivered every TTI (Time Transmission Interval) and $T$ is the subframe duration in seconds. For ATSC 3.0, $T$ is the frame duration. The peak data rate is calculated considering the different system parameters, i.e. CR, modulation order, FFT size, pilot overhead, GI length, preambles L1-basic and L1-detail, bootstrap symbols and frame duration. With ATSC 3.0, $N_{d a t a}$ is calculated as follows:

$$
N_{\text {data }}=N_{F E C}\left(L_{F E C} \cdot R-L_{\text {OUT }}\right)
$$

where $L_{F E C}$ is the FEC block length, $L_{O U T}$ is the number of bits for BCH or CRC if outer coding is used and $N_{F E C}$ is the maximum number of FEC blocks that can be transmitted in a frame. $N_{F E C}$ is in turn calculated as follows:

$$
N_{F E C}=\left\lfloor\frac{N_{\text {cell }} \cdot \log _{2}\left(M_{\text {max }}\right)}{L_{F E C}}\right\rfloor
$$

with $\lfloor\cdot\rfloor$ as the floor function, $N_{\text {cell }}$ as the number of data cells within a frame and $M_{\max }$ as the maximum number of constellation symbols.

\section{B. Peak Spectral Efficiency}

The peak spectral efficiency, expressed in bit $/ \mathrm{s} / \mathrm{Hz}$, is the maximum data rate normalized by carrier bandwidth when excluding radio resources that are used for physical layer synchronization, reference signals or pilots, guard bands and cyclic prefix. The peak spectral efficiency for both LTEAdvanced Pro and ATSC 3.0 can be calculated as:

$$
\eta_{p}=\frac{\gamma_{p}}{B W}
$$

where $B W$ is the bandwidth of the system in $\mathrm{Hz}$, including frequency bands.

\section{Peak BICM Spectral Efficiency}

The peak Bit-Interleaved Coded Modulation (BICM) spectral efficiency defined in bits per channel used (bpc) is the maximum spectral efficiency supported by the system just taking into account cells used for data, i.e. overheads due to synchronization and control channels are not considered. Note that a channel used is directly related to a resource element (RE), defined as a subcarrier in an OFDM signal. This KPI does not depend on the received CNR and therefore it can be calculated through analysis. The peak BICM spectral efficiency is calculated as:

$$
\eta_{p}^{B I C M}=\log _{2}\left(M_{\max }\right) \cdot R_{\max } \cdot N_{T x / R x}
$$

where $R_{\max }$ is the highest efficient CR provided by a particular technology and $N_{T x / R x}$ is the number of independent information spatial streams with multiple transmitter and receiver antennas. Note that the CR in LTE-Advanced Pro is calculated as:

$$
R_{\text {max }}=\frac{N_{\text {data }}}{N_{b}}
$$

where $N_{\text {data }}$ is the number of data bits or TB size, and $N_{b}$ is the number of available bits in a subframe, calculated as:

$$
N_{b}=m \cdot N_{R B}\left(N_{s y m b} N_{s c}^{R B}-N_{r e f}\right)
$$

where $m$ is the number of bits per subcarrier, $N_{R B}$ is the number of resource blocks (RB) utilized within a subframe, $N_{\text {symb }}$ is the number of OFDM symbols per RB dedicated to PTM, $N_{s c}^{R B}$ is the number of subcarriers per RB, and $N_{r e f}$ is the number of subcarriers for reference signals per RB.

\section{BICM Spectral Efficiency}

The BICM spectral efficiency, different from the previous KPI, depends on the received CNR. For the CNR definition used in this paper, the carrier power refers to information carrier power or power in a RE. The BICM spectral efficiency is defined as the number of useful data bits carried in a single RE multiplied by the CR and number of spatial streams necessary to fulfil a particular quality of service (QoS). The QoS is evaluated through link-level simulations. The BICM spectral efficiency is calculated as in (8).

$$
\eta_{B I C M}=\log _{2}(M) \cdot R \cdot N_{T x / R x}
$$

where $M$ is the number of constellation symbols per spatial stream.

\section{E. Mobility}

The last evaluated KPI is mobility, which is defined as the maximum user speed to fulfil a specific QoS. User speeds can be classified in the following items [19]:

- Stationary: $0 \mathrm{~km} / \mathrm{h}$.

- Pedestrian: 0 to $10 \mathrm{~km} / \mathrm{h}$.

- Vehicular: 10 to $120 \mathrm{~km} / \mathrm{h}$.

- High speed vehicular: 120 to $500 \mathrm{~km} / \mathrm{h}$. 


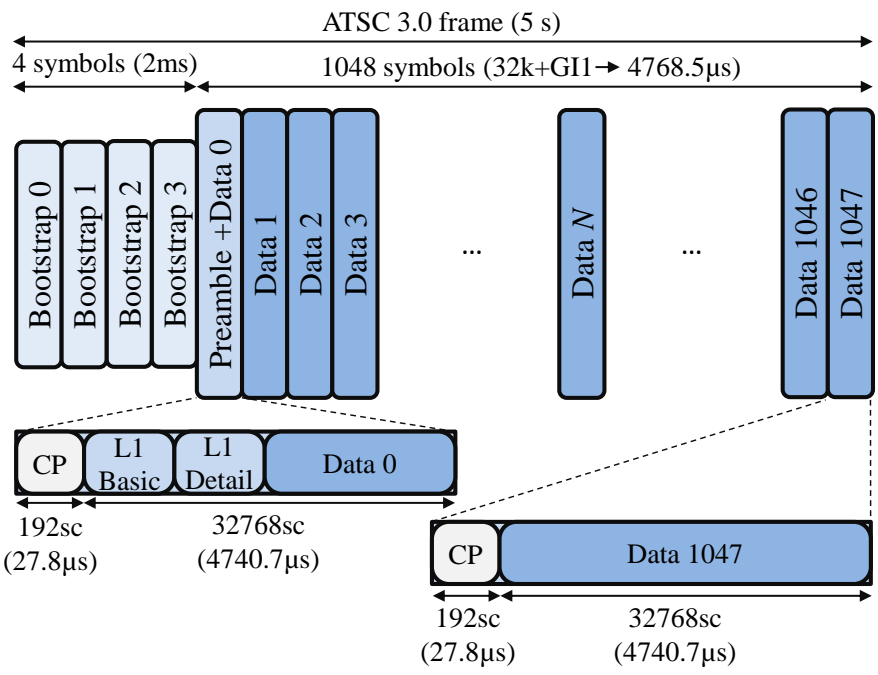

Fig. 2. Maximum number of data cells in the ATSC 3.0 frame.

This work considers the mobility requirement defined in the IMT-2020 recommendation [20], which is set to $250 \mathrm{~km} / \mathrm{h}$ for broadcast and multicast services. Mobility is evaluated through link-level simulations by using a mobile channel model with specific user speed. In mobile environments, a channel realization is a time-variant function that depends on the relative speed of the transmitted and received pair. This time-dependent variation produces a frequency shift at the receiver known as Doppler. The maximum frequency shift $\left(f_{D}\right)$ in $\mathrm{Hz}$ due to the Doppler effect is calculated in (9).

$$
f_{D}=\frac{\nu f_{c} \cos \alpha}{c}
$$

where $\nu$ is the receiver velocity, $f_{c}$ is the signal carrier frequency, $c$ is the speed of light and $\alpha$ is the angle between the receiver direction and the line that connects both transmitter and receiver. In addition, the Doppler limit can be theoretically estimated as [23]:

$$
f_{D_{\text {limit }}}=\frac{1}{2 D_{y}\left(T_{u}+T_{c p}\right)}
$$

where $D_{y}$ is the length of the reference signal sequence in OFDM symbols, $T_{u}$ is the useful symbol duration, and $T_{c p}$ is the cyclic prefix duration. Mobility therefore depends on carrier spacing, bandwidth, frequency band and channel estimation accuracy.

\section{TeChNOlogy AnAlysis}

This section studies the KPIs considered in this work that are based on a theoretical analysis. All KPIs are explored following the methodology described in Section III.

\section{A. Peak Data Rate}

As explained in Section III, the peak data rate takes into account the different overheads due to synchronisation, frequency guard bands, CP, etc. For LTE, this calculation is straightforward, since we only need to know the maximum
TABLE II

ATSC 3.0 PARAMETERS FOR PEAK DATA RATE CALCULATION

\begin{tabular}{cccc}
\hline \hline Parameter & Value & Parameter & Value \\
\hline Frame Duration (sec) & 5 & Bandwidth (MHz) & 6 \\
FFT size & $32 \mathrm{k}$ & $\begin{array}{c}\text { GI (samples) } \\
\text { Boostrap symbol }\end{array}$ & 192 \\
Bootstrap symbols & 4 & duration (ms) & 0.5 \\
Cells in L1-Basic & 163 & Cells in L1-Detail & 922 \\
Pilot Pattern & SP32_2 & Modulation & 4096NUC \\
FEC block (bits) & 64800 & Code Rate & $13 / 15$ \\
\hline \hline
\end{tabular}

TB size transmitted in a single subframe. With SC-PTM, and considering a maximum bandwidth of $20 \mathrm{MHz}$, the maximum TB size or number of data bits $\left(N_{\text {data }}\right)$ is 97896 bits, transmitted in $1 \mathrm{~ms}$. The peak data rate is then calculated using (1), obtaining 97.9 Mbps. Additionally, when considering MIMO for SC-PTM, the peak data rate increases to $195.8 \mathrm{Mbps}$ for $2 \times 2 \mathrm{MIMO}$ and $391.6 \mathrm{Mbps}$ for $4 \times 4$ MIMO. On the other hand, the use of MBSFN limits the peak data rate to 82.6 Mbps. As explained in next subsection, the maximum TB size with MBSFN is limited to 84760 bits, and a CAS has to be transmitted every $40 \mathrm{~ms}$.

For ATSC 3.0, the peak data rate is calculated for the best combination possible, which is illustrated in Table II. Fig. 2 also presents the maximum number of data cells or subcarriers transmitted in a frame $\left(N_{\text {cell }}\right)$. From the 32768 subcarriers shown in Fig. 2, 26113 are active (maximum possible number using a coefficient 4 as specified in [6]). The first data symbol contains 997 pilots (pilot pattern SP32_2), 163 subcarriers for L1-basic and 922 for L1-detail. Therefore, there are 24031 data subcarriers. The 1047 remaining data symbols contain 626 pilots and 27023 data subcarriers. As a consequence, the number of data cells is $N_{\text {data }}=24031+$ $1047 \cdot 27023=28317112$. Since the object of this section is the peak data rate calculation, subframe boundary symbols are not considered. The maximum number of constellation symbols $\left(M_{\max }\right)$ with ATSC 3.0 is 4096 and the highest FEC block length is 64800 bits. From these parameters, the number of FEC blocks $\left(N_{F E C}\right)$ obtained is 5243 , calculated using (3). The maximum number of data bits transmitted and the peak data rate are calculated using (2) and (1) respectively. Considering the same FEC block length of 64800 bits, 13/15 as the maximum CR supported in the LDPC, 192 bits used for $\mathrm{BCH}$ coding and 5 seconds as the frame duration, the resulting peak data rate is $58.70 \mathrm{Mbps}$. This data rate can be doubled if MIMO $2 \times 2$ or channel bonding is used. ATSC 3.0 can provide in both cases up to $117.3 \mathrm{Mbps}$.

\section{B. Peak Spectral Efficiency}

The peak spectral efficiency can be easily calculated using (4). In SC-PTM, the peak data rate is $97.9 \mathrm{Mbps}$ in a maximum bandwidth of $20 \mathrm{MHz}$, resulting in $4.89 \mathrm{bit} / \mathrm{s} / \mathrm{Hz}$. This calculation can be extrapolated to MIMO by modifying the maximum TB size as specified in [21]. Following the same procedure, the peak spectral efficiency with MBSFN is 

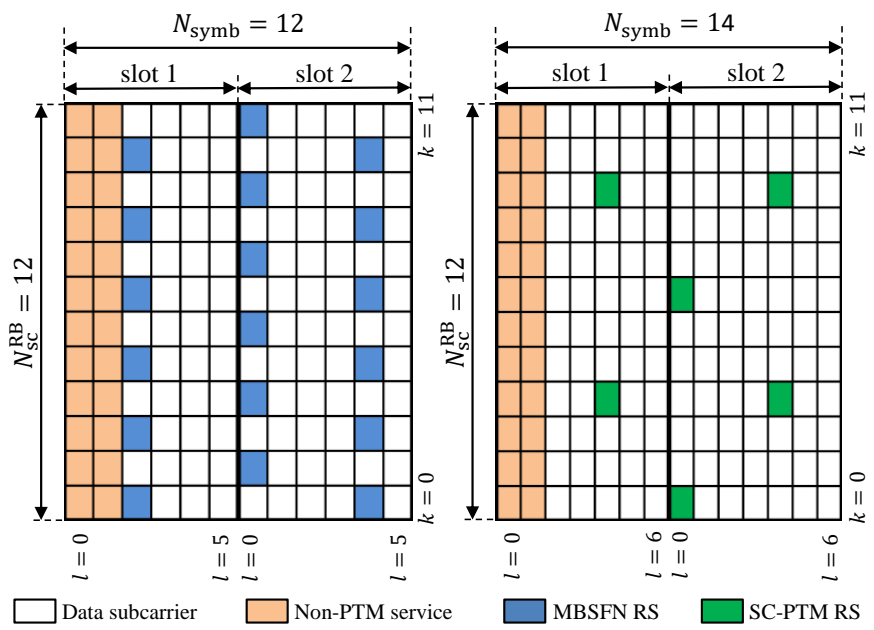

Fig. 3. MBSFN (left) and SC-PTM (right) frame configurations. Both employ a carrier spacing of $15 \mathrm{kHz}$.

TABLE III

SUMMARY OF ATSC 3.0, SC-PTM AND MBSFN ANALYSIS: ANTENNA SCHEME, PEAK BICM SPECTRAL EFFICIENCY, PEAK SPECTRAL EFFICIENCY, OVERHEAD AND PEAK DATA RATE

\begin{tabular}{cccccc}
\hline \hline \multirow{2}{*}{ System } & $\begin{array}{c}\text { Antenna } \\
\text { scheme }\end{array}$ & $\begin{array}{c}\eta_{p}^{B I C M} \\
(\mathrm{bpc})\end{array}$ & $\begin{array}{c}\eta_{p} \\
(\mathrm{bit} / \mathrm{s} / \mathrm{Hz})\end{array}$ & $\begin{array}{c}\text { Overhead } \\
(\%)\end{array}$ & $\begin{array}{c}\gamma_{p} \\
(\mathrm{Mbps})\end{array}$ \\
\hline \multirow{2}{*}{ ATSC 3.0 } & SISO & 10.36 & 9.78 & 5.6 & 58.7 \\
& MIMO 2 $\times 2$ & 20.72 & 19.56 & 5.6 & 117.3 \\
\hline \multirow{3}{*}{ SC-PTM } & SIMO $1 \times 2$ & 7.09 & 4.89 & 30.9 & 97.9 \\
& MIMO 2 $\times 2$ & 14.18 & 9.79 & 30.9 & 195.8 \\
& MIMO 4 $\times 4$ & 28.36 & 19.58 & 30.9 & 391.6 \\
\hline MBSFN & SIMO $1 \times 2$ & 7.06 & 4.13 & 41.5 & 82.6 \\
\hline \hline
\end{tabular}

$4.13 \mathrm{bit} / \mathrm{s} / \mathrm{Hz}$. In ATSC 3.0, the peak data rate with a SingleInput Single-Output (SISO) configuration and without channel bonding is $58.70 \mathrm{Mbps}$, transmitted in $6 \mathrm{MHz}$. In this case, the peak spectral efficiency increases up to $9.78 \mathrm{bit} / \mathrm{s} / \mathrm{Hz}$. If MIMO $2 \times 2$ is taken into account, the value is doubled to $19.56 \mathrm{bit} / \mathrm{s} / \mathrm{Hz}$.

\section{Peak BICM Spectral Efficiency}

As mentioned in previous sections, the peak BICM spectral efficiency depends on the maximum modulation order, effective CR and number of antennas. Fig. 3 shows the different framing configurations for a single $\mathrm{RB}$ and both LTE considered technologies, i.e. SC-PTM and MBSFN. It is noteworthy that the observed carrier spacing is $15 \mathrm{kHz}$ and the number of symbols with MBSFN and SC-PTM is 12 and 14 , due to the use of extended and normal $\mathrm{CP}$ respectively.

In SC-PTM, the number of symbols used for the control channel varies from 1 to 3 [22]. The best option is to use 2 control symbols, since this configuration transmits the highest effective CR $\left(R_{\max }\right)$ that provides the peak BICM data rate as shown in (5). In LTE, $R_{\max }$ is calculated as the TB size $\left(N_{\text {data }}\right)$ divided by the data bits dedicated to PTM within a subframe $\left(N_{b}\right)$, as shown in (6). The use of 1,2 or 3

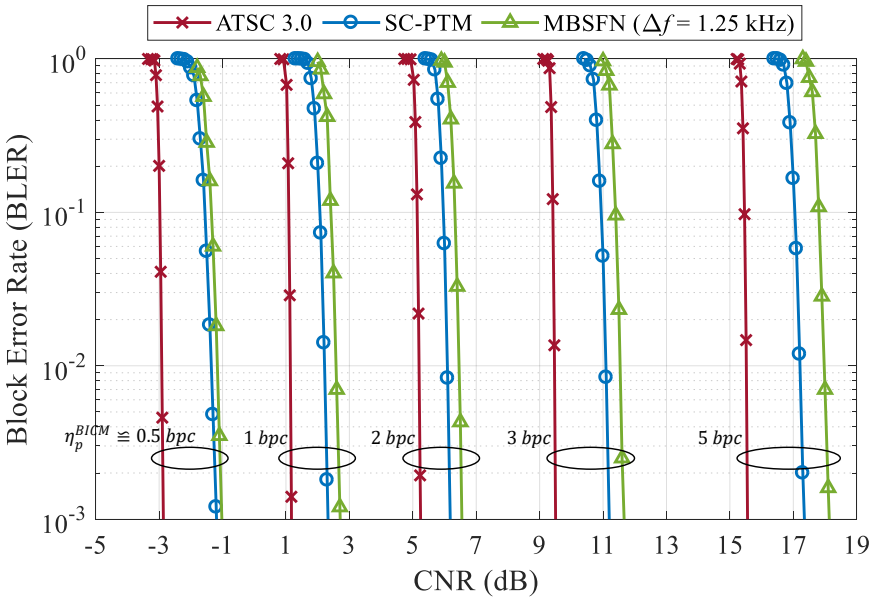

Fig. 4. BLER vs. CNR (dB) for SISO AWGN channel. LTE-Advanced Pro Rel-14 MBSFN, SC-PTM and ATSC 3.0.

control symbols affects the variable $N_{\text {symb }}$ and therefore $N_{b}$. Additionally, $N_{\text {data }}$ may change, since $R_{\max }$ cannot exceed 0.925 , which is the CR associated to the maximum Channel Quality Indicator (CQI) 15 . The maximum number of bits $\left(N_{b}\right)$ with SC-PTM, 2 control symbols and $100 \mathrm{RBs}$, that is, a maximum channel bandwidth of $20 \mathrm{MHz}$, can be calculated using (7), obtaining $8 \cdot 100(12 \cdot 12-6)=110400$ bits. The maximum TB size is given for the index $I_{T B S}=33$ [21] and the associated $\mathrm{CR}$ is then 0.887 .

In MBSFN, the control configuration depends on the selected carrier spacing. In particular, 1 or 2 control symbols are employed with $15 \mathrm{kHz}$, while 7.5 and $1.25 \mathrm{kHz}$ configurations do not dedicate any symbol to control. In this paper, studies for MBSFN are focused on the standalone mode with carrier spacing $1.25 \mathrm{kHz}$. For this configuration, no control symbols are used and the number of bits $N_{b}$ is $8 \cdot 100(1 \cdot 144-24)=96000$. Due to the CR limitation of 0.925 , the maximum TB size is given by the index $I_{T B S}=32$ and the CR is 0.882 .

Since the maximum constellation size is 256QAM, the peak BICM spectral efficiency is 7.09 and 7.06 bpc with SC-PTM and MBSFN respectively. Note that the same calculation can be easily extended to MIMO. SC-PTM with 4 spatial streams (MIMO $4 \times 4$ ) can reach up to $28.36 \mathrm{bpc}$. It is worth pointing out that MBSFN is limited to $7.06 \mathrm{bpc}$ since the use of MIMO is not specified. Without the use of MIMO, ATSC 3.0 provides the highest BICM spectral efficiency with $10.36 \mathrm{bpc}$, due to use of high order constellations with 4096 symbols. ATSC 3.0 supports $2 \times 2 \mathrm{MIMO}$, with a higher peak BICM spectral efficiency of $20.72 \mathrm{bpc}$.

Table III presents a summary of all parameters analyzed in this section. As can be observed, ATSC 3.0, SC-PTM and MBSFN suffer a reduction in spectral efficiency due to overheads of $5.6 \%, 30.9 \%$ and $41.5 \%$ respectively.

\section{Link-Level Performance Evaluation}

The next section evaluates KPIs that are dependent on the network conditions. The required QoS is subject to a block error rate (BLER) lower than 0.1\%. Different scenarios have been evaluated in order to assess the impact of the 
TABLE IV

ATSC 3.0 GAINS FOR REPRESENTATIVE BICM SPECTRAL EFFICIENCIES

\begin{tabular}{ccccc}
\hline \hline $\begin{array}{c}\eta_{B I C M} \\
(\mathrm{bpc})\end{array}$ & $\begin{array}{c}\text { CNR ATSC 3.0 } \\
(\mathrm{dB})\end{array}$ & $\begin{array}{c}\text { NUC gain } \\
(\mathrm{dB})\end{array}$ & $\begin{array}{c}\text { Gain over } \\
\text { MBSFN }(\mathrm{dB})\end{array}$ & $\begin{array}{c}\text { Gain over } \\
\text { SC-PTM }(\mathrm{dB})\end{array}$ \\
\hline 0.5 & -2.9 & - & 1.8 & 1.7 \\
1 & 1.1 & - & 1.6 & 1.2 \\
2 & 5.1 & 0.2 & 1.1 & 1.4 \\
3 & 9.5 & 0.5 & 2.1 & 1.7 \\
5 & 15.6 & 1 & 2.5 & 1.8 \\
\hline \hline
\end{tabular}

configurations adopted. A bandwidth of $10 \mathrm{MHz}$ has been used with both LTE configurations and a subcarrier spacing of $\Delta_{f}=1.25 \mathrm{kHz}$ is always used with MBSFN. This assumption is taken in order to study the potential advantages of this mode compared to SC-PTM.

\section{A. BICM Spectral Efficiency}

1) Additive White Gaussian Noise (AWGN) Channel: Fig. 4 shows the performance for representative spectral efficiencies of SC-PTM, MBSFN and ATSC 3.0 of approximately $0.5,1$, 2, 3 and $5 \mathrm{bpc}$, in an AWGN channel. With LTE-Advance Pro PTM technologies, MCS used are 4, 8, 13, 19 and 27 (Table 7.1.7.1-1 in [21]) respectively. Note that provided BICM spectral efficiencies are in fact slightly different due to overheads and control symbols. In this case, a single antenna is considered in both transmitter and receiver. As depicted in Fig. 4, ATSC 3.0 provides important gains compared to LTE. Table IV provides the CNR gains for selected values. It is important to highlight that the LTE results are obtained with a sub-optimum turbo-decoder that reduces complexity at the expense of a performance loss.

The use of NUCs for modulation and LDPC codes in ATSC 3.0 achieves high performance gains. NUCs provide a significant improvement due to the geometrical signal shaping and increases with the constellation order. Table IV also shows different NUC gains obtained for ATSC 3.0 and BICM spectral efficiencies of 2, 3 and $5 \mathrm{bpc}$ [24]. The use of NUCs can reduce the required CNR up to $1 \mathrm{~dB}$ with 256QAM modulations. As main drawback, NUCs adopted in ATSC 3.0 for this modulation order increase the demapping complexity at the receiver, since they do not have square shape and therefore inphase and quadrature components cannot be separated. Note that no gains are obtained for 0.5 and $1 \mathrm{bpc}$. The constellation used is QPSK, which does not permit any optimization. The ATSC 3.0 performance depends highly on the codeword length, with 64800 bits providing higher gains compared to 16200 bits. This gain also depends on the CR, achieving from $0.2 \mathrm{~dB}$ (high $\mathrm{CR}$ ) to $0.7 \mathrm{~dB}$ (robust $\mathrm{CR}$ ), regardless of the modulation order.

2) i.i.d. Rayleigh MIMO channel: Fig. 5 (top left) shows the BICM spectral efficiency achieved with the different technologies evaluated, for the independent and identically distributed (i.i.d.) Rayleigh MIMO channel. The channel capacity is also shown for comparison. Whereas ATSC 3.0 and SC-PTM utilize 2 transmitter and receiver antennas, MBSFN employs a
$1 \times 2$ Single-Input Multiple-Output (SIMO) scheme, which is a major drawback. An ideal cross-polar channel is used, with no depolarization between both transmitted streams. A Minimum Mean Square Error (MMSE) demapper has been used with ATSC 3.0 and SC-PTM in order to cope with computation complexity limitations. Modulation and coding combinations with poor performance have been removed.

The use of multiple transmit and receive antennas can provide important spatial multiplexing gains at high CNRs. Although MBSFN is limited to $7 \mathrm{bpc}$, SC-PTM and ATSC 3.0 can increase their limits to more than $12 \mathrm{bpc}$. Comparing ATSC 3.0 and SC-PTM, the former provides better performance. At a low spectral efficiency of $1 \mathrm{bpc}$, ATSC 3.0 and SC-PTM provide similar performance. However, for higher BICM spectral efficiencies, ATSC 3.0 clearly outperforms SCPTM. This is mainly due to the use of longer codewords, LDPC codes and NUCs in ATSC 3.0. Note that the architectures employed in both LTE-Advanced Pro and ATSC 3.0 systems for this work are similar. ATSC 3.0 uses a single codeword that is then multiplexed over the two transmitter antennas. LTE has been also simulated with a single codeword that is mapped to the two layers using the second option in Table 6.3.3.2-1 [22] and two antennas ports using precoding without cyclic delay diversity.

3) Fixed-Rooftop Reception: Fig. 5 (top right) depicts the BICM spectral efficiency for the DVB-F1 channel model [23], which is commonly used to model fixed-rooftop reception conditions. As a consequence of the channel characteristics, a performance degradation is introduced compared to AWGN channel. In particular, a CNR increase between 0.1 and $0.7 \mathrm{~dB}$ is observed, depending on the used configuration. In addition, ATSC 3.0 provides even higher gains than those observed in AWGN channel. This is due to the additional frequency diversity provided by the frequency interleaver.

A CNR value commonly used to model fixed rooftop reception in real scenarios is $20 \mathrm{~dB}$. For the considered CNR, ATSC 3.0 provides a BICM spectral efficiency of $6.1 \mathrm{bpc}$. LTEAdvanced Pro, on the other hand, transmits $5.3 \mathrm{bpc}$ (regardless of the PTM technology used) while requiring the same CNR. The ATSC 3.0 capacity gain for this scenario is therefore 0.8 bpc. The overall performance with LTE-Advanced Pro follows the same trend regardless of the PTM technology used and the different carrier spacing values. The use of a higher carrier spacing of $15 \mathrm{kHz}$ instead of $1.25 \mathrm{kHz}$ does modify the CR and therefore the required CNR to achieve 0.1\% BLER but it also changes the BICM spectral efficiency achieved.

4) Portable-Outdoor Reception: The NGH - Portable Outdoor (NGH-PO) channel [25] models static reception in outdoor environments. Due to a strong line of sight, it exhibits low frequency selectivity. Fig. 5 (bottom left) shows the performance of the different PTM technologies evaluated. Because of the high computational burden that this channel model entails (a large number of channel snapshots need to be simulated to obtain statistically reliable results), a representative set of LTE MCS indexes and ATSC 3.0 modulation and coding configurations is selected.

A CNR value commonly used to model portable outdoor reception is $10 \mathrm{~dB}$. In this particular case, ATSC 3.0 provides 

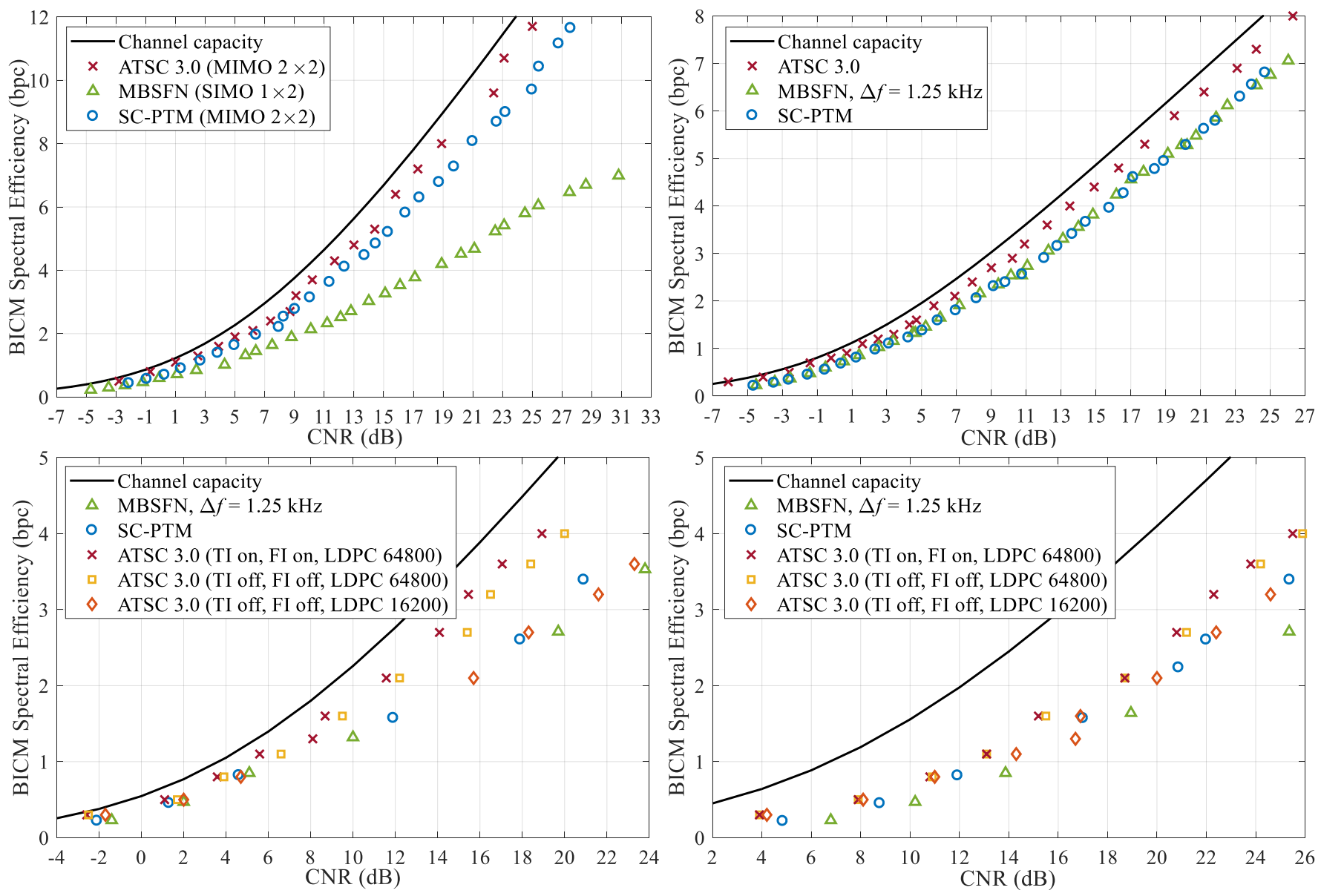

Fig. 5. BICM spectral efficiency vs. CNR in dB of LTE-Advanced Pro Rel'14 MBSFN, SC-PTM and ATSC 3.0. Different channel models are evaluated: i.i.d. Rayleigh MIMO channel (top left), DVB-F1 Rice (top right), NGH-PO (bottom left) and NGH-PI (bottom right).

a BICM spectral efficiency of 1.9 bpc. On the other hand, $1.4 \mathrm{bpc}$ is obtained with SC-PTM while MBSFN provides a slightly higher value of $1.6 \mathrm{bpc}$. The ATSC 3.0 capacity gain when using $100 \mathrm{~ms}$ of TIL for this scenario is therefore 0.5 and $0.3 \mathrm{bpc}$ compared to SC-PTM and MBSFN respectively.

ATSC 3.0 provides higher gains than in previous scenarios. This performance gain comes from the two additional time and frequency interleavers. When none of these interleavers are used, ATSC 3.0 performance depends on the LDPC code length. If a long LDPC code length of 64800 bits is kept, the performance gets $1-2 \mathrm{~dB}$ worse than for cases with time and/or frequency interleaving. For short LDPC length, CNR degradations between 1 and $2 \mathrm{~dB}$ are obtained for robust configurations, while high-capacity configurations have from 5 to $7 \mathrm{~dB}$ of loss. Overall, PTM technologies with short codewords, such as MBSFN or ATSC with 16200 bits, suffer significant performance degradation.

5) Portable-Indoor Reception: In this scenario, NGH Portable Indoor (NGH-PI) [25] models static reception at indoor environments where the multipath effect implies a higher frequency selectivity compared to the outdoor scenarios. Fig. 5 (bottom right) shows the performance of the different PTM technologies for this scenario. It can be observed that the channel capacity gap has been increased compared to NGH-
PO. This is due to a higher cross-polarization discrimination factor, which reduces the direct channel component power. Whereas NGH-PO is modelled with a factor 4, NGH-PI is defined with 1.78. In addition, the difference between both LTE-Advanced Pro technologies becomes larger with the CNR. The use of higher CRs combined with the use of a narrow carrier spacing degrades the performance significantly with MBSFN. For instance, the difference in CNR for the MCS 33 (256QAM, CR 0.85) is higher than $5 \mathrm{~dB}$.

When using ATSC 3.0 without time and frequency interleaving, the CNR depends again on the LDPC code length. For NGH-PI, this code length variation has a lower impact in the performance compared to the NGH-PO channel, as a consequence of the power reduction of the direct channel component. However, an LDPC code length of 16200 bits deteriorates the CNR up to $3 \mathrm{~dB}$, compared to a code length 64000. It can be also observed that the effect of interleavers in this case is not significant.

\section{B. Mobility Evaluation}

In order to evaluate the mobility, the 6-tap Typical Urban (TU-6) channel model is considered [26]. To mitigate Doppler shift in mobile scenarios, ATSC 3.0 employs a convolutional TIL with different TIL depths assumed for a Single Physi- 


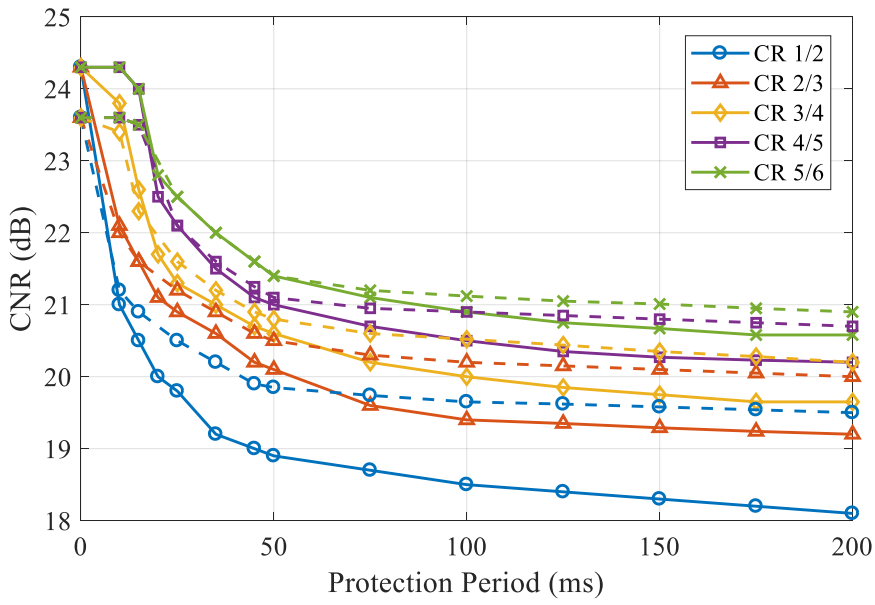

Fig. 6. CNR vs. protection period in low diversity (dashed lines) and in high diversity (solid lines) scenarios for MBSFN with MCS 21.

cal Layer Pipe (S-PLP) mode. The convolutional TIL depth depends on the number of convolutional rows, i.e. 0,512 , 724, and 1024, which represent approximate TIL depths of $\Delta T=0,50,100$, and $200 \mathrm{~ms}$, respectively [27]. Since in MBSFN standalone mode $\left(\Delta_{f}=1.25 \mathrm{kHz}\right)$ a single OFDM symbol occupies the whole RB, no TIL at physical layer can be applied. Instead, the use of forward error correction at application layer (AL-FEC) is evaluated.

1) Use of AL-FEC Codes in MBSFN Signals: AL-FEC mechanisms are used to recover packet losses derived from underlying layers, allowing the correction of end-to-end errors in scenarios with considerable time variability, caused mainly by fast fading and shadowing effects. The AL-FEC coding process is defined by three parameters: the protection period $\left(T_{p}\right)$ measured in ms, which is the time interleaving depth achieved at the application layer; the code-rate; and the source symbol size $\left(T_{s}\right)$ measured in bytes. AL-FEC encoding in eMBMS is based on Raptor codes. Note that ideal AL-FEC coding has been considered in this paper for the sake of simplicity.

Assuming $T_{s}$ equal to the TB size, FEC blocks are created and grouped in order to constitute Internet Protocol (IP) packets of 1024 bytes. The CR determines the number of erroneous IP packets that can be corrected. Lower CRs increase ALFEC protection against errors but also increase the overhead. The protection period fixes the time length for source blocks transmission and is selected depending on the desired delay and memory available at the device. Longer protection periods take advantage of the temporal diversity but also increase the end-to-end delay and zapping time, which has an impact on the QoS.

In Fig. 6, the AL-FEC performance is compared in two different scenarios, low and high temporal diversity, considering different CRs and protection period values. For low diversity, the NGH-PO channel with speed $3 \mathrm{~km} / \mathrm{h}$ is used, at $700 \mathrm{MHz}$ carrier frequency. High diversity is represented via a TU-6 mobile channel with $120 \mathrm{~km} / \mathrm{h}$ at $700 \mathrm{MHz}$ carrier frequency. As can be observed, AL-FEC coding provides an important gain in mobile scenarios with time variability, especially using

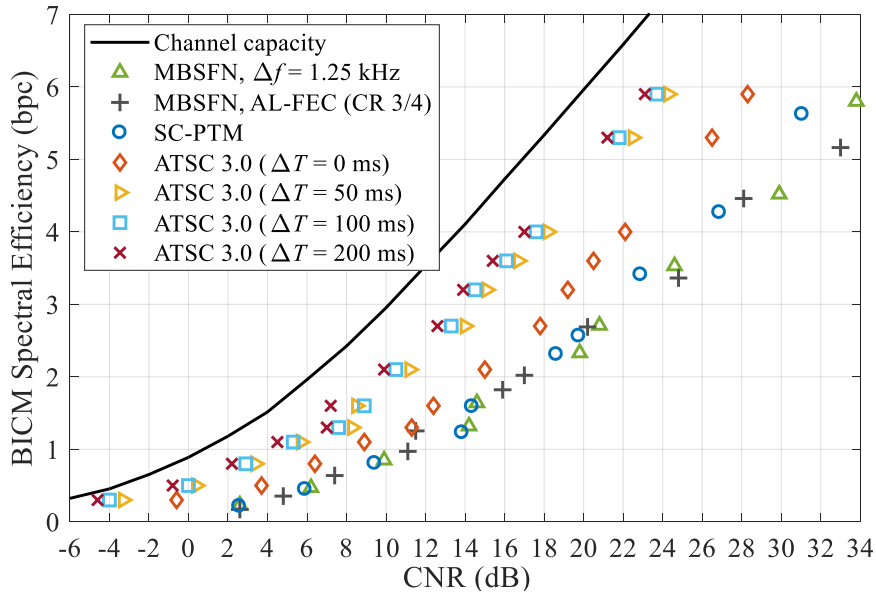

Fig. 7. BICM spectral efficiency vs. CNR in dB of LTE-Advanced Pro Rel14 MBSFN, SC-PTM and ATSC 3.0. TU-6 mobile channel with $120 \mathrm{~km} / \mathrm{h}$ at $700 \mathrm{MHz}$ carrier frequency.

robust CRs and when the protection period is long enough. However, AL-FEC is not efficient in scenarios with fixed channels due to the lack of time diversity, regardless of the configuration used.

2) Vehicular reception: As mentioned before, the use of time interleaving at the physical layer in ATSC 3.0 can provide significant gains compared to eMBMS. Results in Fig. 7 demonstrate that gains obtained are always high, regardless of the modulation order and $\mathrm{CR}$ used. Gains from 4 to 5 $\mathrm{dB}$ appear when using the maximum depth of $\Delta T=200 \mathrm{~ms}$. However, a TIL depth of $\Delta T=50 \mathrm{~ms}$ is sufficient to achieve important gains in a wide range of spectral efficiencies. From these results, it can be concluded that short TIL in eMBMS could be applied for these mobility scenarios, although this approach would require interleaving of more than one subframe. Comparing both LTE-Advanced Pro PTM solutions, SC-PTM outperforms MBSFN. The use a larger carrier spacing makes easier the demodulation despite the Doppler shift introduced by the channel.

MBSFN with AL-FEC is also evaluated in this case, with configuration parameters $\mathrm{CR} 3 / 4$ and $T_{p}=100 \mathrm{~ms}$. ALFEC MBSFN can improve the reception for mobile channels with time diversity when there is no TIL at the physical layer. At high CNRs, some MBSFN AL-FEC cases provide better performance than simple MBSFN cases, but AL-FEC gains become negligible at low CNRs. Some alternatives like moving down AL-FEC to link or physical layer could improve the performance in terms of latency and robustness, at the expense of increased memory requirements at the receiver [28].

3) Speed Resilience with Practical Receiving Algorithms: This subsection evaluates the use of PTM solutions for a wide range of Doppler shifts at the frequency band of $700 \mathrm{MHz}$. Only ATSC 3.0 and MBSFN with carrier spacing $1.25 \mathrm{kHz}$ have been considered in this study as corner cases, since SCPTM uses carrier spacing $15 \mathrm{kHz}$, which is less limiting in this particular scenario. Narrow subcarrier spacings are more susceptible of experimenting Inter-Carrier Interference (ICI) 

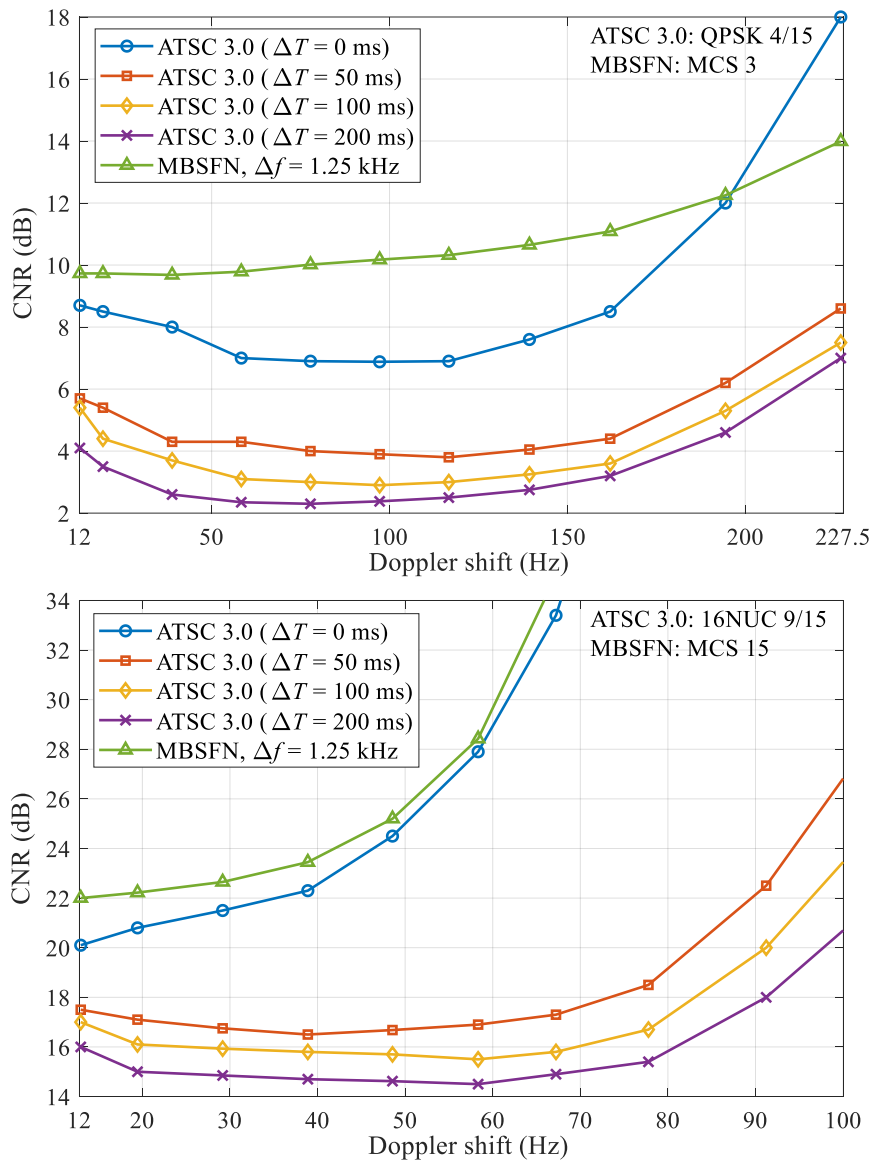

Fig. 8. CNR to achieve a BLER $0.1 \%$ against user speed, for SC-PTM, MBSFN and ATSC 3.0 in TU-6 mobile channel. MCS 3 vs. QPSK 4/15 (top) and MCS 15 vs. 16NUC 9/15 (bottom).

in mobile environments due to the Doppler shift introduced. In fact, the theoretical Doppler limits of each PTM technology can be calculated using (10). The relationship between Doppler shift and speed for both technologies considered can be calculated using (9). To that end, it is necessary to know that $T_{U}+T_{C P}=1407.67 \mu$ s for ATSC 3.0 and $1 \mathrm{~ms}$ for MBSFN. Therefore, the Doppler limit in ATSC 3.0 for the configuration selected is $177.6 \mathrm{~Hz}(275 \mathrm{~km} / \mathrm{h})$, while the limit for MBSFN is $250 \mathrm{~Hz}(385 \mathrm{~km} / \mathrm{h})$.

Without the use of time interleaving, the theoretical Doppler limit can only be achieved if the selected MCS is robust enough. In mobility simulations, the Doppler limit is calculated as the value that entails a CNR performance loss of $3 \mathrm{~dB}$ compared to the lowest CNR achieved in the whole range [29]. Fig. 8 (top) shows the required CNR with different Doppler shifts and real channel estimation, for MCS 3 with MBSFN and QPSK 4/15 with ATSC 3.0. The estimation is formed by a Least Square (LS) estimator for reference signals, followed by a 2D linear interpolation applied in time and frequency domains. Results show that for Doppler shifts up to $150 \mathrm{~Hz}$ (user speeds of $225 \mathrm{~km} / \mathrm{h}$ ), the performance with both technologies is good enough and the CNR is maintained. However, for higher user speeds, the Doppler shift starts to cause significant ICI and channel estimation errors. The limits with MBSFN and ATSC 3.0 are 210 and $175 \mathrm{~Hz}$ respectively, very close to the theoretical values.

The Doppler limit is drastically decreased in Fig. 8 (bottom), since a less robust MCS 15 is used. ATSC 3.0 employs an equivalent configuration of $16 \mathrm{NUC} 9 / 15$. In this case, the permitted Doppler shifts are only 47 and $45 \mathrm{~Hz}$ with MBSFN and ATSC 3.0 respectively (the Doppler range shown at the bottom is lower than the range at the top). The only way to increase the limits is by using a time interleaver, as observed for ATSC 3.0. The TIL always decreases the CNR regardless of the selected modulation and CR and the user speed under evaluation.

\section{CONCLUSION}

In this paper, the state of the art PTM technologies, i.e. ATSC 3.0 and the two LTE-Advanced Pro variants MBSFN and SC-PTM, have been evaluated through analysis and linklevel simulations. These results will serve as a benchmark to compare the performance of a potential $5 \mathrm{G}$ broadcast solution. The presented analysis has revealed that without the use of MIMO, ATSC 3.0 provides the highest BICM spectral efficiency $(10.36 \mathrm{bpc})$, while SC-PTM is the best option for MIMO with 4 spatial streams $(28.36 \mathrm{bpc})$. Regarding peak data rate, ATSC 3.0 is able to deliver 117.3 Mbps with $2 \times 2$ MIMO, while SC-PTM can deliver up to $391.6 \mathrm{Mbps}$ (in one RF carrier) with $4 \times 4$ MIMO. MBSFN does not support MIMO, and therefore the peak data rate is limited to 82.6 Mbps.

Link-level results in this paper have shown that the use of long codewords with LDPC codes in ATSC 3.0 provides significant gains at the expense of longer latencies. In addition, the use of non-uniform constellations can provide CNR gains up to $1 \mathrm{~dB}$ but with an increased demapping complexity as main drawback. The use of multiple transmit and receive antennas achieves spatial multiplexing gains at high CNR values. Regarding mobility, the use of time interleaving at the physical layer in ATSC 3.0 can provide significant gains compared to LTE-Advanced Pro for time variant scenarios. In addition, SC-PTM outperforms MBSFN $\left(\Delta_{f}=1.25 \mathrm{kHz}\right)$ in this case. To enhance the resilience of MBSFN transmissions, AL-FEC could be adopted at the expense of reducing the spectral efficiency and increasing the zapping time. As an alternative, the use of time interleaving can also increase the maximum speed that mobile users can tolerate without significant performance degradation.

\section{REFERENCES}

[1] G. A. Reitmeier and T. R. Smith, "An Overview of the ATSC Digital Television Standard," in International Workshop on HDTV, Los Angeles, USA, Oct. 1996.

[2] M. Takada and M. Saito, "Transmission System for ISDB-T," Proceedings of the IEEE, vol. 94, no. 1, pp. 251-256, Jan. 2006.

[3] J. Song et al., "Technical Review on Chinese Digital Terrestrial Television Broadcasting Standard and Measurements on Some Working Modes," IEEE Trans. on Broadcast.g, vol. 53, no. 1, pp. 1-7, March 2007.

[4] U. Ladebusch and C. A. Liss, "Terrestrial DVB (DVB-T): A Broadcast Technology for Stationary Portable and Mobile Use," Proceedings of the IEEE, vol. 94, no. 1, pp. 183-193, Jan. 2006.

[5] I. Eizmendi et al., "DVB-T2: The Second Generation of Terrestrial Digital Video Broadcasting System," IEEE Trans. on Broadcast., vol. 60, no. 2, pp. 258-271, June 2014. 
[6] L. Fay et al., "An Overview of the ATSC 3.0 Physical Layer Specification," IEEE Trans. on Broadcast., vol. 62, no. 1, pp. 159-171, March 2016.

[7] G. Faria, J.A. Henriksson, E. Stare and P. Talmola, "DVB-H: Digital Broadcast Services to Handheld Devices," Proceedings of the IEEE, vol. 94, no. 1, pp. 194-209, Jan. 2006.

[8] D. Gomez-Barquero, C. Douillard, P. Moss and V. Mignone, "DVBNGH: The Next Generation of Digital Broadcast Services to Handheld Devices," IEEE Trans. on Broadcast., vol. 60, no. 2, pp. 246-257, June 2014.

[9] D. Lecompte and F. Gabin, "Evolved Multimedia Broadcast/Multicast Service (eMBMS) in LTE-Advanced: Overview and Rel-11 Enhancements," IEEE Communications Magazine, vol. 50, no. 11, pp. 68-74, Nov. 2012.

[10] D. Vargas and D. Mi, Eds., "LTE-Advanced Pro Broadcast Radio Access Network Benchmark," Deliverable D3.1, 5G-PPP 5G-Xcast project, Nov. 2017.

[11] H. Chen et al., "Pioneering Studies on LTE eMBMS: Towards 5G Pointto-Multipoint Transmissions," IEEE Sensor Array and Multichannel Signal Processing (SAM) Workshop, Sheffield, UK, July 2018.

[12] International Telecommunications Union (ITU), Draft New Report ITUR M., "Guidelines for evaluation of radio interface technologies for IMT-2020," Oct. 2017.

[13] 3GPP TS 22.261 v16.3.0, "Service requirements for the 5G system; Stage 1 (Release 16)," Mar. 2018.

[14] D. Gomez-Barquero, D. Navratil, S. Appleby and M. Stagg, "Point-toMultipoint Communication Enablers for the Fifth-Generation of Wireless Systems”, IEEE Communications Standards Magazine, vol. 2, no. 1, pp. 53-59, March 2018.

[15] K.-J. Kim et al., "Low-Density Parity-Check Codes for ATSC 3.0," IEEE Trans. on Broadcast., vol. 62, no. 1, pp. 189-196 , March 2016.

[16] L. Michael and D. Gomez-Barquero, "Bit-Interleaved Coded Modulation (BICM) for ATSC 3.0," IEEE Trans. on Broadcast., vol. 62, no. 1, pp. 181-188, March 2016.

[17] Advanced Television Systems Committee, Document A/322:2017, "Standard: Physical Layer Protocol," June 2017.

[18] International Telecommunications Union (ITU), Draft New Report ITUR M., "Minimum requirements related to technical performance for IMT2020 radio interface(s)," Feb. 2017

[19] D. Ratkaj and A. Murphy, Eds., "Definition of Use Cases, Requirements and KPIs," Deliverable D2.1, 5G-PPP 5G-Xcast project, Oct. 2017.

[20] 3GPP TR 38.913 v14.3.0, "Study on scenarios and requirements for next generation access technologies," June 2017.

[21] 3GPP TS 36.213 v14.2.0, "Evolved Universal Terrestrial Radio Access (E-UTRA); Physical layer procedures (Release 14)," Mar. 2017.

[22] 3GPP TS 36.211 v14.2.0, "Evolved Universal Terrestrial Radio Access (E-UTRA); Physical channels and modulation (Release 14)," Mar. 2017.

[23] ETSI TR 102831 v1.1.0, "Implementation guidelines for a second generation digital terrestrial television broadcasting system (DVB-T2)," Oct. 2010.

[24] N. Loghin et al., "Non-Uniform Constellations for ATSC 3.0," IEEE Trans. on Broadcast., vol. 62, no. 1, pp. 197-203, Mar. 2016.

[25] P. Moss, T. Y. Poon and J. Boyer, BBC White Paper WHP 205, "A simple model of the UHF cross-polar terrestrial channel for DVB-NGH," Sep. 2011.

[26] ETSI TR 102401 v1.1.1, "Digital Video Broadcasting (DVB); Transmission to Handheld Terminals (DVB-H); Validation Task Force Report," May 2005

[27] P. Klenner et al., "Physical Layer Time Interleaving for the ATSC 3.0 System," IEEE Trans. on Broadcast., vol. 62, no. 1, pp. 253-262, March 2016.

[28] D. Gomez-Barquero et al., "A Novel Physical Layer Split FEC Scheme for Long Time Interleaving With Fast Zapping Support," IEEE Trans. on Broadcast., vol. 58, no. 2, pp. 269-276, June 2012.

[29] S. I. Park et al., "Low Complexity Layered Division Multiplexing for ATSC 3.0," IEEE Trans. on Broadcast., vol. 62, no. 1, pp. 233-243, March 2016.

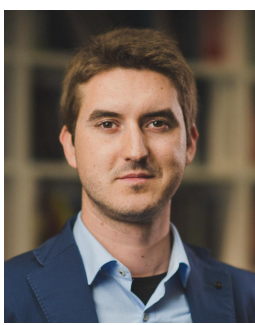

Manuel Fuentes received his M.Sc. degree in telecommunication engineering and a second M.Sc. degree in communication technologies, systems and networks from the Universitat Politecnica de Valencia, Spain, in 2012 and 2013 respectively. He also obtained the Ph.D. degree in telecommunication engineering in 2017. Currently, he is working at the Institute of Telecommunications and Multimedia Applications (iTEAM). He has been a guest researcher at the Vienna University of Technology, Austria, in 2016. He has also contributed actively to the ATSC 3.0 standardization process. In 2017, Dr. Fuentes joined the Samsung Electronics R\&D UK team as a $5 \mathrm{G}$ research engineer to participate for one year in the 5P-PPP phase-2 project 5G-Xcast, for the efficient delivery of broadcasting in 5G New Radio systems. His main research interests include physical layer procedures, innovative techniques in bit-interleaved coding and modulation such as non-uniform constellations or signal space diversity, and multi-antenna communications.

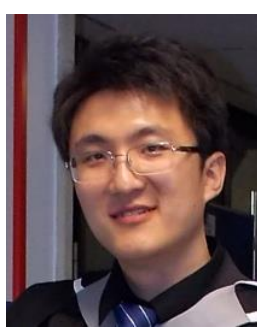

De Mi received the B.Eng. degree in Information Engineering from Beijing Institute of Technology, Beijing, China, in 2011. He then received the M.Sc. degree in Communications and Signal Processing from Imperial College London, UK, in 2012. In 2017 he received his Ph.D. degree from the Institute for Communications Systems, home of the 5G Innovation Centre, University of Surrey, UK, where he is currently a research fellow in Wireless Communications. His research interests include air interface design, multi-antenna signal processing, broadcast and multicast transmissions, and millimetre-wave communications.

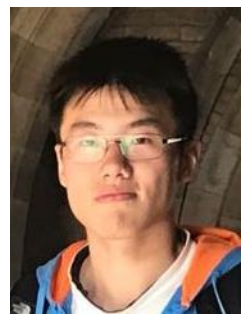

Hongzhi Chen received the B.Eng. degree in Northumbria University, Newcastle upon Tyne, UK, in 2014 and the M.Sc. degree in Mobile and Satellite Communication from University of Surrey, UK, in 2015. He is currently a Ph.D. candidate in the Institute for Communications Systems, home of the 5G Innovation Centre, University of Surrey, UK. His research interests include air interface design for point to point and point to multipoint transmissions and convex optimizations.

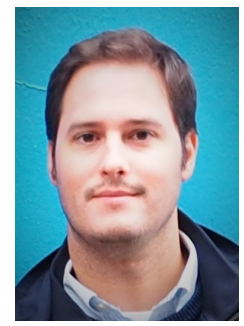

Eduardo Garro is a R\&D engineer at Mobile Communications Group (MCG) of the Institute of Telecommunications and Multimedia Applications (iTEAM) at Universitat Politecnica de Valencia (UPV). He received a M.Sc. degree in Telecommunications engineering and a second M.Sc. degree in Communications and Development of Mobile Services from UPV, Spain in 2013 and 2014 respectively. In 2012, he joined the iTEAM, working with Agencia Nacional del Espectro (ANE), the spectrum regulator of Colombia on the coexistence between DTT and 4G (LTE) technologies. He has also participated on the planning and optimization of DVB-T2 networks in Colombia. He has been also involved in the standardization of ATSC 3.0. He is currently pursuing his Ph.D. degree in terrestrial broadcasting and is involved in the 5G-Xcast (Broadcast \& Multicast Communication Enablers for the Fifth-Generation of Wireless Systems) project. His research activities are focused on Non-Orthogonal Multiple Access (NOMA), multiple antenna systems (MIMO), and realistic channel estimation methods in broadcasting networks. 


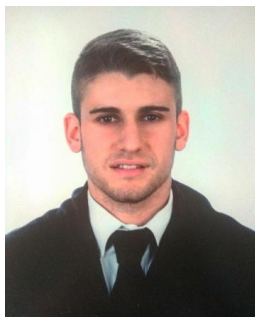

Jose Luis Carcel received the B.Sc. degree in Telecommunications engineering from the Universitat Politecnica de Valencia (UPV) in 2016. He is currently a M.Sc. student at UPV and $5 \mathrm{G}$ researcher at Samsung Electronics R\&D UK. His current research activities are included within the $5 \mathrm{G}-\mathrm{PPP}$ project $5 \mathrm{G}-\mathrm{X}$ cast, where he is contributing to the study of physical layer mechanisms for a $5 \mathrm{G}$ NR broadcast solution. His main research interests include the study of new 5G numerologies and candidate waveforms to operate in Single Frequency Networks (SFNs). His research on Null-Prefix OFDM operation was awarded with the "2017 Best Student Paper Award" at IEEE International Symposium on Broadband Multimedia Systems and Broadcasting (Cagliary, Italy).

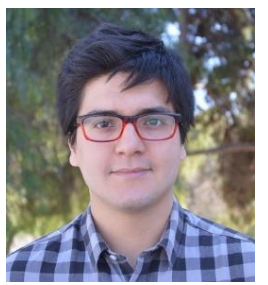

David Vargas is a Project Research Engineer at the BBC R\&D, London, UK. He received a M. Sc. degree in Telecommunications engineering, a M. Sc. degree in Technologies, Systems and Communication Networks, and a Ph.D. degree in Telecommunications from Universitat Politecnica de Valencia (UPV), Spain in 2009, 2011 and 2016, respectively. $\mathrm{He}$ has been a guest researcher at the Vienna University of Technology, Vienna, Austria and at McGill University, Montreal, Canada. David has experience in standardization of technical specifications such as the mobile broadcasting standard DVB-NGH and the terrestrial broadcasting standard ATSC 3.0. He has also been delegate in various 3GPP working groups representing the $\mathrm{BBC}$. His research interests include multi-antenna communications, signal processing for communications, wireless communications and digital broadcasting.

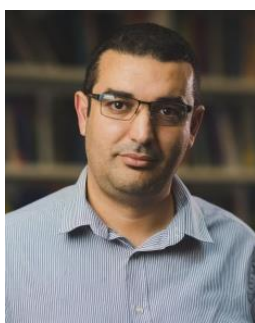

Belkacem Mouhouche received his Ph.D. degree in Signal Processing from Ecole Nationale Superieure des Telecoms (Telecom Paristech) in France in 2005. During his Ph.D he worked on 3GPP third generation systems (UMTS), he later worked as a 3GPP standard Engineer following the development of HSDPA and LTE systems. He was also involved in FP7 European Project End to End Efficiency (E3) where he worked on cognitive radio in heterogeneous systems. Before joining Samsung he worked in major telecommunication companies like Sierra Wireless, Freescale Semiconductor, NEC and Alcatel Lucent. His research interest are in the area of advanced techniques for the physical layer of future communication systems including MIMO, beamforming and coding applied to broadcast and broadband systems like the LTE-A and DVB, ATSC3.0. Recently, He was heavily involved in the development of the latest broadcast terrestrial standard ATSC 3.0 and lead the broadcast activity within the 5GPPP project Fantastic-5G. Recently, he was appointed as innovation and dissemination manager of the 5G-PPP project 5G-Xcast focused on new point to multipoint techniques for $5 \mathrm{G}$ networks.

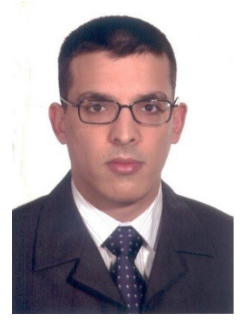

David Gomez-Barquero received a Ph.D. degree in telecommunications engineering from the Universitat Politecnica de Valencia (UPV), Spain. He is a senior researcher (Ramon \& Cajal Fellow) at UPV's Institute of Telecommunications and Multimedia Applications (iTEAM), where he leads a research group working on next-generation wireless broadcast technologies. Dr. Gomez-Barquero has been during the last decade actively participating in the digital television standardization, including DVB-T2, T2-Lite, DVB-NGH, and ATSC 3.0. His recent research interests are focused in the development of broadcast and multicast technology components for 5G. He is the project manager of the 5GPPP project 5G-Xcast. He is an Associate Editor of the IEEE TRANSACTIONS ON BROADCASTING. 